\title{
Developmental Venous Anomaly Presenting Intracranial Hemorrhage without Associated Vascular Anomaly
}

\author{
Sung Ho Kim, Dong Gyu Yeo, Gwang Su Lee \\ Department of Neurosurgery, Soonchunhyang University Gumi Hospital, Gumi, Korea
}

\begin{abstract}
Developmental venous anomalies (DVAs) are cited as the most common intracranial vascular anomaly. In the majority of cases, it has a benign clinical course, and complications such as intracranial hemorrhage (ICH) are rarely reported. In the rare cases of DVAs presenting $\mathrm{ICH}$, the causes of hemorrhage are usually by a combined vascular anomaly, mostly cavernous malformation (CM) or complicated DVAs, such as thrombosis in the vein of DVAs. We report a rare case of uncomplicated DVA presenting hemorrhage without accompanied vascular anomaly, such as CM. The cause of hemorrhage is suggested to be a specific form of DVA, arterialized DVA. Arterialized DVAs should be noted that they have a greater risk of hemorrhage than classic DVAs.
\end{abstract}

Keywords: Developmental venous anomaly; Intracranial hemorrhages; Arterialized developmental venous anomaly

\section{INTRODUCTION}

Cerebral vascular malformations are classified into capillary telangiectasia, cavernous malformation (CM), arteriovenous malformation (AVM), and developmental venous anomaly (DVA) [1]. DVAs are cited as the most common cerebral vascular anomaly and in the majority of cases, DVAs follow a benign clinical course. Hemorrhagic risk of DVA is low and the causes of hemorrhage are usually by a combined vascular anomaly, mostly CM or complicated DVAs, such as thrombosis in the vein of DVAs. We report a rare case of uncomplicated DVA presenting hemorrhage without accompanied vascular anomaly, such as CM.

\section{CASE REPORT}

A 5-year-old female presented to the emergency room with a stuporous mentality after a sudden generalized tonic-clonic type seizure attack 2 hours before admission. The patient had no seizure history and no other past medical history. Since the patient was a child with a first seizure attack, initially enhance brain computed tomography (CT) was performed, demonstrating left basal ganglia intracranial hemorrhage (ICH) with subarachnoid hemorrhage in the left Sylvian fissure and basal cistern, and some calcified lesion adjacent to the hematoma (Fig. 1A-D). On enhancement, there were multiple small and giant enhancing vessels, suggesting a vascular anomaly, DVA, or AVM with draining veins (Fig. 1E). There was herniation of the brain by the mass effect of $\mathrm{ICH}$ and planned for decompressive craniectomy and hematoma removal. Before surgery, we needed to evaluate the vascular anomaly, so performed CT angiography showing a DVA with a giant collector vein, however, there was an ICH and there were multiple fistulous connections with artery and veins, so we could not rule out AVM (Fig. 2). The patient was in an emergent situation for decompressive craniectomy due to high intracranial pressure, so a thorough evaluation of the vascular anomaly such as conventional angiography could not be done. The operation was performed emergently just to decompress the brain by wide craniectomy and careful evacuation of the hematoma not injuring the malformed vessels. Postoperatively the brain was well decompressed (Fig. 3), and the patient improved significantly but had right-sided hemiparesis (grade 4) with clumsiness. At about 1 month after the operation, magnetic resonance imaging was performed and there
Correspondence to: Sung Ho Kim

Department of Neurosurgery, Soonchunhyang University Gumi Hospital, 179 1(il)gongdan-ro, Gumi 39371, Korea

Tel: +82-54-468-9132, Fax: +82-54-468-9075, E-mail: 68512@schmc.ac.kr

Received: May 11, 2021 / Accepted after revision: Jun. 14, 2021
(C) 2021 Soonchunhyang Medical Research Institute

This is an Open Access article distributed under the terms of the Creative Commons Attribution Non-Commercial License (https://creativecommons.org/licenses/by-nc/4.0/) 

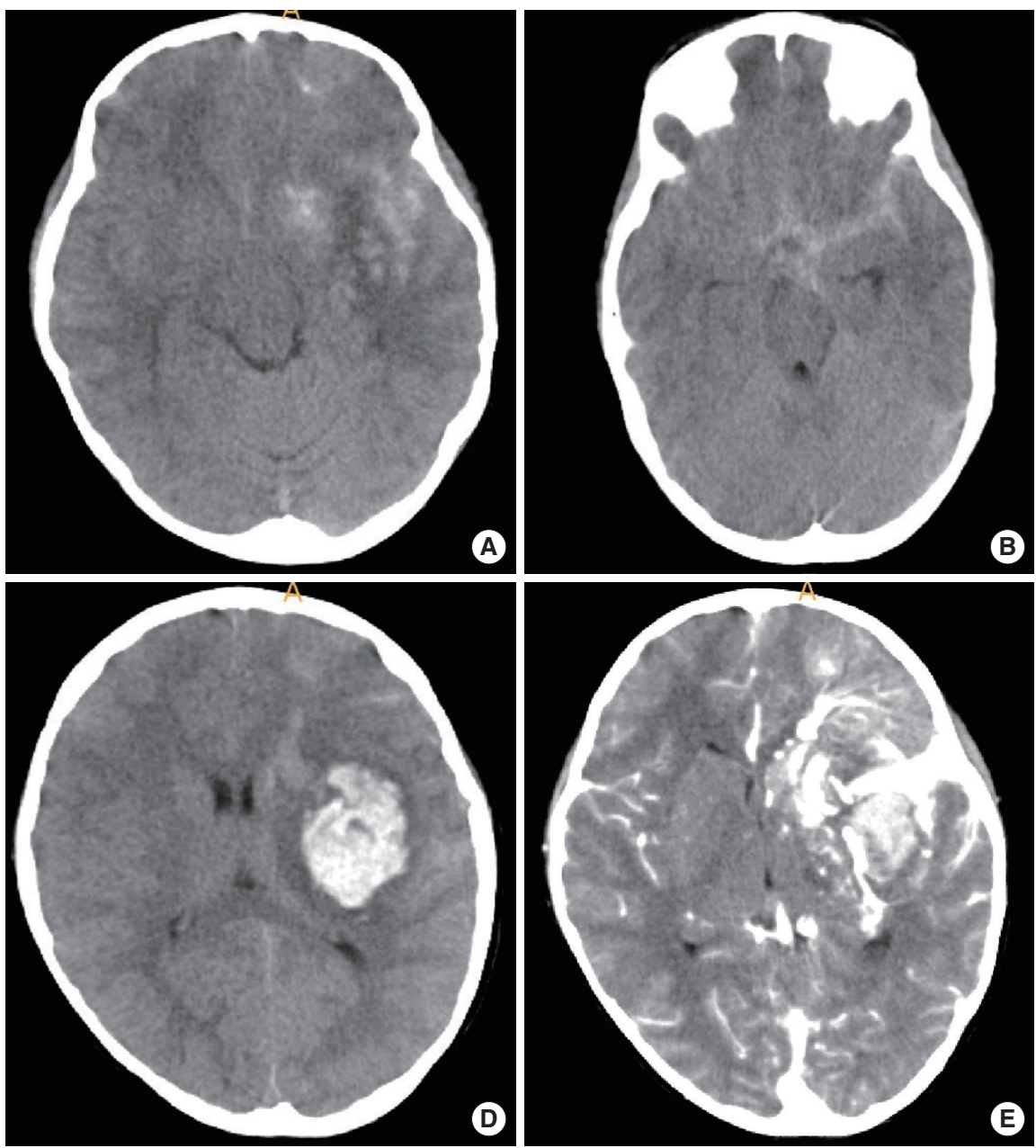

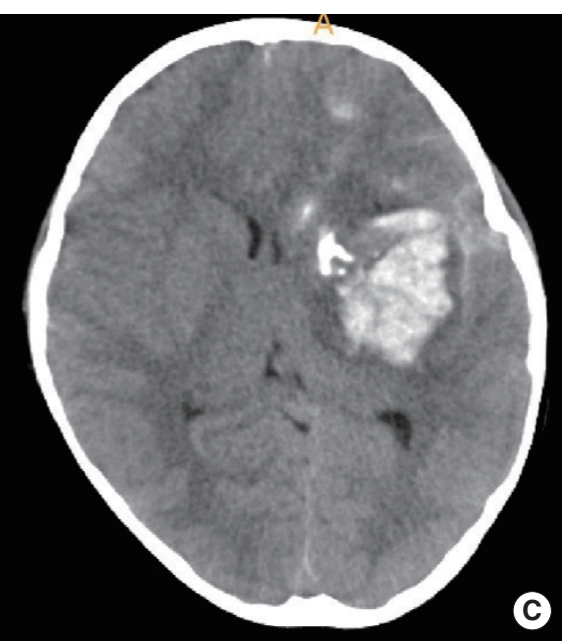

Fig. 1. (A-D) A 5-year-old female presented to the emergency room by stuporous mentality after sudden generalized tonic-clonic type seizure attack. Initial brain computed tomography (B-CT) demonstrating left basal ganglia intracranial hemorrhage with subarachnoid hemorrhage in the left Sylvian fissure and basal cistern, and some calcified lesion adjacent to the hematoma (arrow). (E) On B-CT enhancement, there were multiple small and giant enhancing vessel, suggesting a vascular anomaly, developmental venous anomaly, or arteriovenous malformation with draining veins.

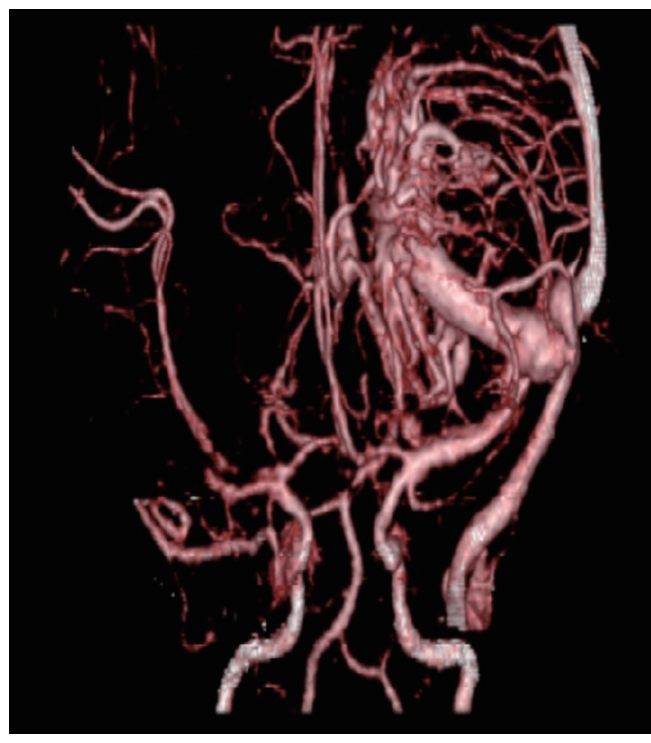

Fig. 2. Brain computed tomography angiography was performed showing a developmental venous anomaly with a giant collector vein with multiple fistulous connection with artery and veins.

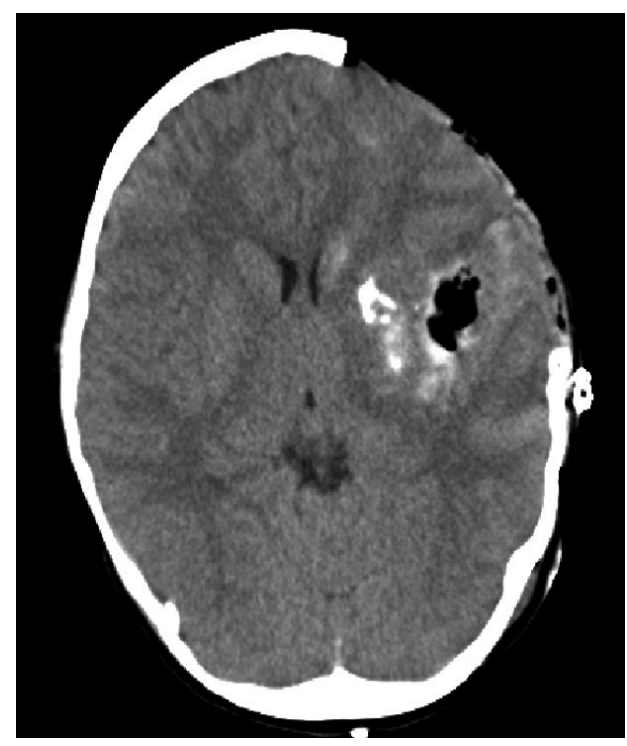

Fig. 3. Postoperative brain computed tomography showing subtotal intracranial hemorrhage removal and the brain is well decompressed. 
Kim SH, et al. • DVA Presenting ICH without Associated Vascular Anomaly

were no other specific findings besides the lesion shown on the initial workups, such as associated vascular anomaly or lesion suggesting thrombosis of DVA (Fig. 4A-D). A conventional angiogram was planned but the patient's parent wanted further work up in a tertiary hospital, and conventional angiogram was performed
5 months later at a tertiary hospital. The angiogram showed an arterialized DVA, DVA appearing in the late arterial phase, it was accompanied by numerous arteriovenous communications with capillary blush without a demonstrable arterial feeder or AVM nidus (Fig. 5A-H). Cranioplasty was performed and the patient is in
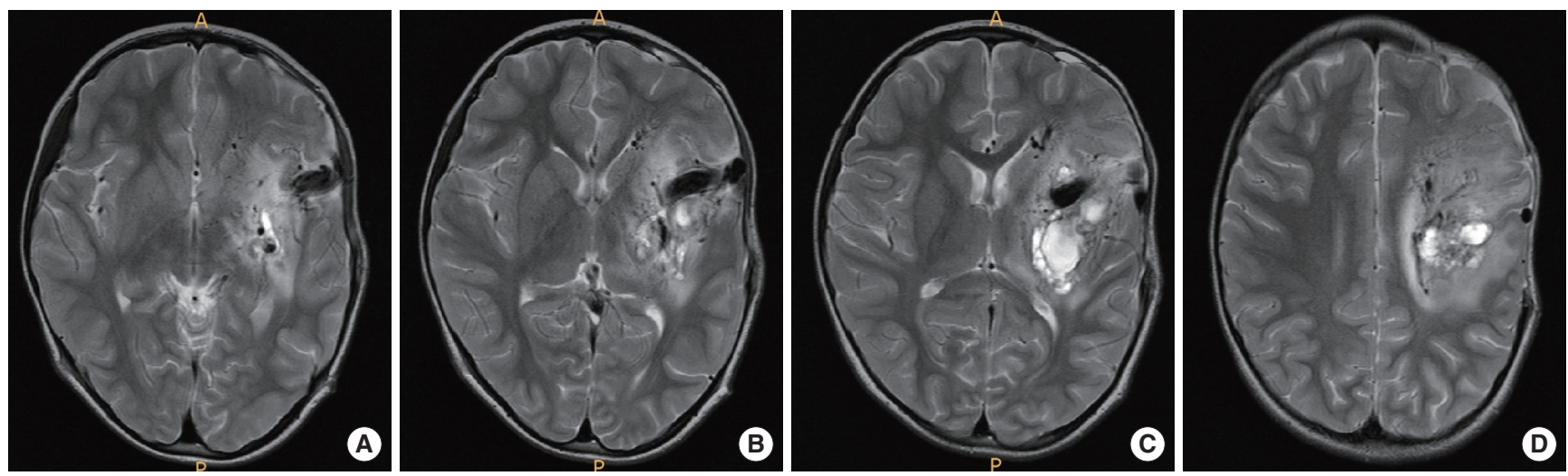

Fig. 4. (A-D) After about 1 month after operation, brain magnetic resonance imaging was performed, and there were no other specific findings beside the lesion shown on initial workup, a giant signal void suggesting collector vein of developmental venous anomaly.
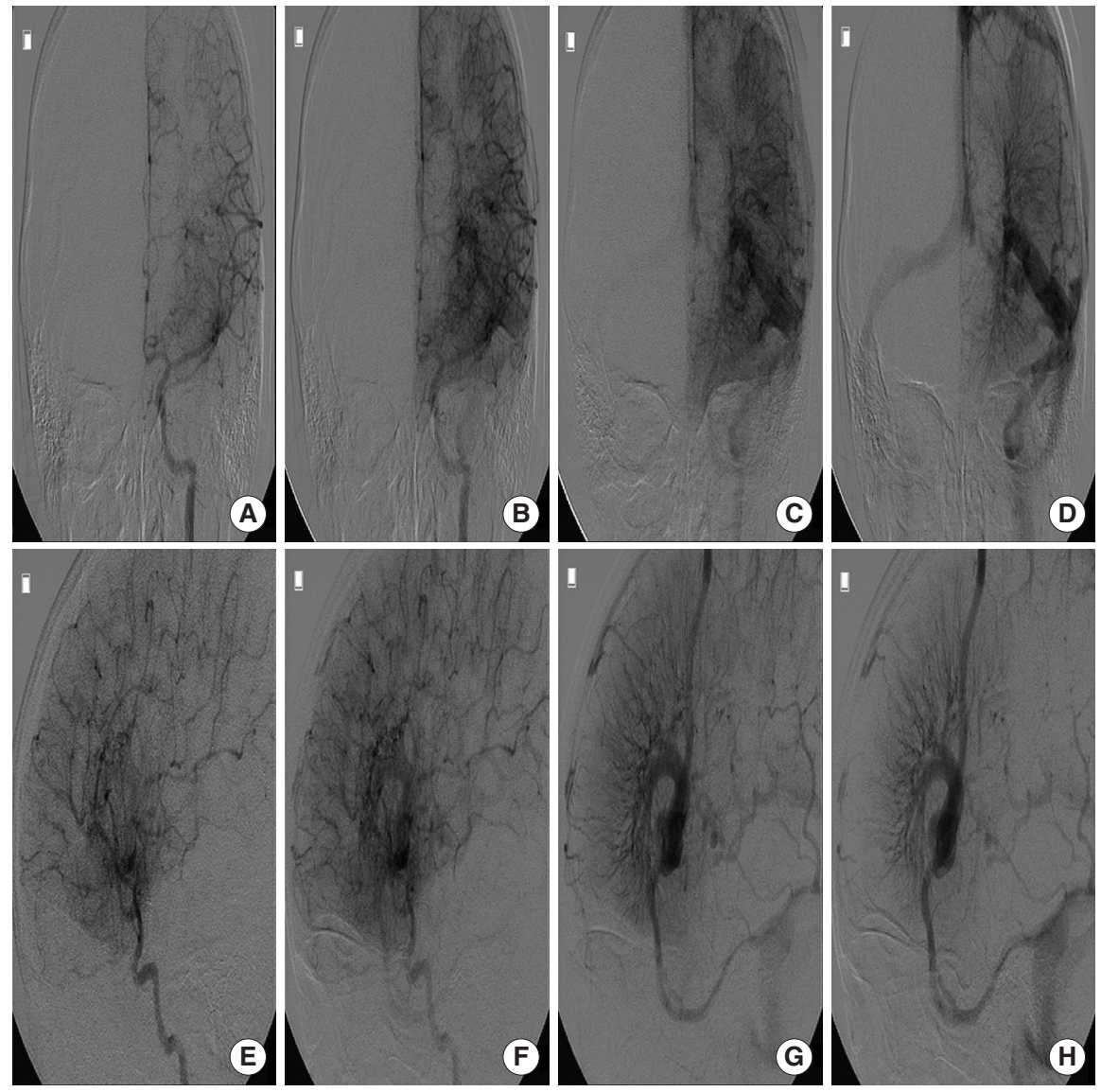

Fig. 5. (A-H) At 2 months after the operation, a conventional angiogram was performed showing a developmental venous anomaly, appearing in the late arterial phase accompanied by numerous arteriovenous communications with capillary blush without a demonstratable arterial feeder or arteriovenous malformation nidus. 
DVA Presenting ICH without Associated Vascular Anomaly • Kim SH, et al.

good condition, performing well in school. There were a few seizures attack after surgery, so the patient is on anticonvulsants in an outpatient clinic.

The patient's caregiver provided written informed consent for the publication of clinical details and images.

\section{DISCUSSION}

Cerebral vascular malformations are classified into capillary telangiectasia, CM, AVM, and DVA [1]. DVAs are cited as the most common cerebral vascular anomaly, reported up to $2.6 \%$ in a series of 4,069 brain autopsies [2]. The defining feature of DVA is the confluence of radially oriented veins into a single dilated venous channel. Although there is no consensus to explain the pathogenic mechanism of development of DVAs, it is presumed DVAs are formed during intrauterine life by the alteration in venous drainage due to either thrombotic occlusion or arrested development of the venous system in the fetal development. To compensate for the change of venous drainage, locoregional parenchymal veins join into a large collecting single vein, forming the hallmark of DVAs, 'caput medusae'. The term "developmental venous anomaly" proposed by Lasjaunias et al. [3] is now widely used as a synonym for cerebral venous angiomas, cerebral venous malformations, and cerebral venous medullary malformations, which emphasize the nature of DVAs to be a variant of venous drainage rather than a true vascular anomaly. It should be noted that DVAs represent normal venous drainage of a normal brain, and sacrificing DVA can lead to venous infarction in the draining territories.

Although DVAs are mostly found as an isolated incidental lesion, they can be associated with adjacent pathology. The most common associated lesion is cerebral CM. DVAs are associated with sporadic CMs in $13 \%$ to $40 \%$ of cases [2] and when CMs are associated with DVAs, the CMs are located adjacent to DVAs. This common association and the close topographic relation suggest DVAs are responsible in formation of CMs, and reports of de novo formation of $\mathrm{CM}$ in a patient with DVA support this assumption [2,4]. Venous congestion and venous hypertension within DVAs are demonstrated in the literature and subclinical microhemorrhages in a form of macrophage laden hemosiderin surrounding a DVA are reported, so it can be hypothesized that diapedesis through the wall of the venous radicles or rupture of one of the radicles of the DVA may occur which induces activation of angiogenic growth factors, such as vascular endothelial growth factor, leading to $\mathrm{CM}$ formation
$[2,4]$. Sinus pericranii (SPi), abnormal communication between extracranial veins and intracranial dural sinuses, is another concomitant lesion well known. Gandolfo et al. [5] reported DVA was found in seven out of 15 patients with SPi, and there were cases of SPi being the major drainage pathway of the DVA. This emphasizes the need for thorough exploration for concomitant DVA when planning treatment of SPi, since the closure of DVA will induce venous infarction. DVAs also have been reported with increased incidence in patients with superficial venous malformations of the head and neck, lymphatic malformations of the orbit and periorbital region, and gastrointestinal tract (blue rubber bleb nevus syndrome [2]. Incidentally found abnormalities of the brain parenchyme surrounding DVAs, such as locoregional cerebral atrophy, dystrophic calcification, and nonspecific white matter lesions are reported, thought to be secondary to venous hypertension by DVAs [2].

In the majority of cases, DVAs follow a benign clinical course. Clinical presentations are usually caused by complicated DVAs, such as thrombotic occlusion of the collecting vein, or by the accompanied vascular anomaly, mostly CM or AVM, arteriovenous fistula (AVF). Hemorrhagic risk of DVA is low, considered $<0.7 \%$, and the cause of hemorrhage is usually the accompanied vascular anomaly, mostly CM [1]. Only a few reports have cited DVA without $\mathrm{CM}$ as the cause of $\mathrm{ICH}$, and these authors suggest an associated AVM or AVF, venous thrombosis of DVA as the cause of hemorrhage $[6,7]$. Venous thrombosis can also induce ischemic venous infarction or reversible cerebral edema around a DVA other than ICH producing neurological deficit [2]. Agarwal et al. [6] has reported a unique case of $\mathrm{ICH}$ in a patient with DVA, the cause of hemorrhage to be a vasculitis, which was proved by histologic evaluation.

Hemorrhage in the presence of DVA without accompanied vascular anomaly, especially CM or thrombosis of DVA is very rare. Here we report a rare case of DVA itself, arterialized DVA, producing hemorrhage. In this case accompanied vascular anomaly or thrombosis of DVA were excluded by radiologic evaluations, and on conventional angiography, arterialized DVA was to be the cause of hemorrhage. Arterialized DVA is defined when DVA appeared on an angiogram in the arterial phase, accompanied by numerous arteriovenous communications with capillary blush [8]. Oran et al. [8] reported that only 53 cases of arterialized DVA were reported in the literature and at least 19 cases (36\%) were associated with $\mathrm{ICH}$, hemorrhage appears to be more frequent in arterial- 
Kim SH, et al. • DVA Presenting ICH without Associated Vascular Anomaly

ized DVA than in the cases of classic DVA. Ruiz et al. [4] proposed three types of arterialized DVA. Type 1 as typical DVA occurring during mid or late arterial phases, without demonstrable arterial feeders or AVM nidus, and type 2 as "arterialized DVA" with enlarged arterial feeders to the caput medusa, without demonstrable AVM nidus which was identical with this case (Fig. 5A, B). Type 3 refers to DVA draining an angiographically demonstrable AVM. Arterialized DVA especially types 2 and 3 should be noted that they seem to have similar clinical behavior with AVM which has a greater risk to develop hemorrhage than classic DVAs [2].

The pathophysiology of arterialized DVA is unknown. Several assumptions are made. Lasjaunias et al. [3] reported DVAs are thought to have a lesser adaptive capacity than a normal venous structure that produces arteriovenous shunts, AVM, or CM. Another report by Mullan et al. [9] proposed a theory that AVMs and DVAs have a common origin related to a defective formation of the venous mantel, and arterialized DVAs are a transitional form between DVA and AVM. Awad et al. [10] suggested that arterialized DVAs are a mixed form of vascular malformation of a DVA and AVM. Management of strategy of these arterialized DVAs is not established due to insufficient data. However, there are a few case reports or small series published so far, which reports types 2 and 3 arterialized DVA presenting hemorrhage have been treated by various combinations of endovascular embolization, in particular, when AVM nidus was demonstrated, surgical resection, and radiosurgery, that treatment plan to be aggressive as AVM. And some authors recommend conservative treatment [2].

We report a rare case of uncomplicated DVA presenting hemorrhage without accompanied vascular anomaly. The cause of hemorrhage is suggested to be a specific form of DVA, arterialized DVA. Arterialized DVAs should be noted that they have a greater risk of hemorrhage than classic DVAs, seem to have similar clini- cal behavior with AVM, and though treatment strategy is not well established, should be followed up with great care.

\section{CONFLICT OF INTEREST}

No potential conflict of interest relevant to this article was reported.

\section{REFERENCES}

1. Idiculla PS, Gurala D, Philipose J, Rajdev K, Patibandla P. Cerebral cavernous malformations, developmental venous anomaly, and its coexistence: a review. Eur Neurol 2020;83:360-8.

2. San Millan Ruiz D, Gailloud P. Cerebral developmental venous anomalies. Childs Nerv Syst 2010;26:1395-406.

3. Lasjaunias P, Burrows P, Planet C. Developmental venous anomalies (DVA): the so-called venous angioma. Neurosurg Rev 1986;9:233-42.

4. Ruíz DS, Yilmaz H, Gailloud P. Cerebral developmental venous anomalies: current concepts. Ann Neurol 2009;66:271-83.

5. Gandolfo C, Krings T, Alvarez H, Ozanne A, Schaaf M, Baccin CE, et al. Sinus pericranii: diagnostic and therapeutic considerations in 15 patients. Neuroradiology 2007;49:505-14.

6. Agarwal N, Zuccoli G, Murdoch G, Jankowitz BT, Greene S. Developmental venous anomaly presenting as a spontaneous intraparenchymal hematoma without thrombosis. Neuroradiol J 2016;29:465-9.

7. Roh JE, Cha SH, Lee SY, Jeon MH, Cho BS, Kang MH, et al. Atypical developmental venous anomaly associated with single arteriovenous fistula and intracerebral hemorrhage: a case demonstrated by superselective angiography. Korean J Radiol 2012;13:107-10.

8. Oran I, Kiroglu Y, Yurt A, Ozer FD, Acar F, Dalbasti T, et al. Developmental venous anomaly (DVA) with arterial component: a rare cause of intracranial haemorrhage. Neuroradiology 2009;51:25-32.

9. Mullan S, Mojtahedi S, Johnson DL, Macdonald RL. Cerebral venous malformation-arteriovenous malformation transition forms. J Neurosurg 1996;85:9-13.

10. Awad IA, Robinson JR Jr, Mohanty S, Estes ML. Mixed vascular malformations of the brain: clinical and pathogenetic considerations. Neurosurgery 1993;33:179-88. 